\title{
A Study on Applications and Case Studies Regarding Bluetooth Technology for Travel Time Measurement
}

\author{
Dian Yuan, Ardeshir Faghri, Katherine Partridge \\ Department of Civil and Environmental Engineering, The University of Delaware, Newark, DE, USA \\ Email: diany@udel.edu,faghri@udel.edu,kpartridge@udel.edu
}

How to cite this paper: Yuan, D., Faghri, A. and Partridge, K. (2020) A Study on Applications and Case Studies Regarding Bluetooth Technology for Travel Time Measurement. Journal of Transportation Technologies, 10, 65-87.

https://doi.org/10.4236/jtts.2020.101005

Received: December 20, 2019

Accepted: January 10, 2020

Published: January 13, 2020

Copyright ( 2020 by author(s) and Scientific Research Publishing Inc. This work is licensed under the Creative Commons Attribution International License (CC BY 4.0).

http://creativecommons.org/licenses/by/4.0/

\begin{abstract}
Bluetooth technology emerged over twenty years ago and has continuously improved throughout the years to meet diverse and complex applications. Initially invented to replace the need for physical data cables, Bluetooth offers users a quick and easy way to share data files over a wireless network. Traffic engineers and transportation engineering researchers have utilized the potential opportunities that exist with Bluetooth and have implemented this technology into traffic monitoring techniques. To gain a better understanding of Bluetooth sensors and how they work, a comprehensive literature search was conducted. Twenty-five articles were studied regarding case studies of Bluetooth sensor implementation for travel time measurement. Besides reviewing the literature and previous case studies, three new case studies in the State of Delaware, USA, were also conducted and carefully analyzed. The benefits and drawbacks associated with Bluetooth technology for travel time measurements have been identified in this paper. The overall conclusion of the authors is Bluetooth alone and by itself is not a proper technology for travel time measurements. More studies need to be conducted on the accuracy and overall application, before one can confidently utilize the Bluetooth technology for travel time measurements.
\end{abstract}

\section{Keywords}

Travel Time Measurement, Bluetooth Technology, Comparison, Applications, Literature Review, Case Studies

\section{Background and Introduction}

Bluetooth technology is a wireless alternative that allows users to interconnect various electronics (e.g., cellphones, laptops, computers, speakers/headsets) to 
one another and share data over this connection. Created in 1994, the purpose of Bluetooth was to establish a connection and collaboration between unique products and industries. The concept of Bluetooth is to be ideally utilized over short-range distances [1]. The application of Bluetooth technology contains hardware and software components. Bluetooth hardware consists of an antenna of some sort to transmit signals from one device to another; the software is effectively responsible for interpreting radio signals to and from the device [2]. The radio frequency that Bluetooth commonly operates at is $2.4 \mathrm{GHz}$. When present among other devices that utilize a radio frequency to operate, the Bluetooth transmitted signal "hops around the frequency". This phenomenon is called Frequency Hopping Spread Spectrum (FHSS). In order to establish a connection between two Bluetooth devices, the two devices must be "paired" [1].

Bluetooth devices can be broken down into three general classes-Class I, Class II, and Class III, referring to the power that is transmitted from the devices. Table 1 provides a general overview of the three different power classes that Bluetooth devices are commonly described by. For pairing two devices from different classes, the maximum communicating distance is based on the one with shorter range [3].

Bluetooth technology has been innovated throughout the years since it was first introduced to the public over twenty years ago. It was primarily created to eliminate the need for data cables and connections. This technology has made wireless keyboards, mice, and even wireless printing possible [4]. Over the years, this technology has been greatly improved and led to simplicity in other applications. Bluetooth is commonly recognized and associated with communication. Extending Bluetooth technology to mobile phones has led to hands-free communication. Such applications have guided Bluetooth technology to be implemented in cars. Vehicles equipped with Bluetooth technology allow the driver to make hands-free calls and even texts while driving on the road. Another very common application of Bluetooth is wireless networking. Because Bluetooth interconnects two electronic devices, data or information that can be transferred, shared, and synchronized over this wireless connection. A more recent application of Bluetooth technology is travel time measurement. This application will be further discussed in this paper.

The Bluetooth Special Interest Group (SIG) owns the Bluetooth trademark [1]. The Bluetooth SIG licenses out the trademark to companies who have earned membership within the SIG [1]. Generally, the Bluetooth devices are powered by private companies. Bluetooth sensors have been deployed in different locations all over the United States, Canada, Australia, New Zealand, and countries in Europe to collect travel time data. The travel time data collected by these Bluetooth sensors is sometimes used to produce origin-destination matrices for freeways, arterials, and urban roadways. Additionally, these sensors are also used to monitor real-time traffic conditions. Different models of Bluetooth sensors are available for different applications. 
Table 1. Bluetooth device power classes. Source: SANS Technology Institute [3].

\begin{tabular}{cccc}
\hline Class & $\begin{array}{c}\text { Maximum Transmitted } \\
\text { Power (milli-Watts) }\end{array}$ & $\begin{array}{c}\text { Maximum Range } \\
\text { (meters) }\end{array}$ & Applications \\
\hline I & 100 & 100 & Laptops, Desktops \\
II & 2.5 & 10 & Mobile phones, Headsets \\
III & 1 & $<10$ & Bluetooth adapters \\
\hline
\end{tabular}

Although Bluetooth is quick to install and easily adaptable, there are some risks that are associated with the technology. Security breaches are one of the main concerns associated with this technology. Eavesdropping is a possible concern when Bluetooth headsets are used. Despite the many iterations and improvements with this technology, hackers tend to always find a flaw in the software [5]. Furthermore, whenever data is transferred across a Bluetooth connection, users are highly advised to properly encrypt the data. Failure to encrypt the data properly may result in interception or even disclosure of the transferred data by the hacker [6]. Bluetooth connections in automobiles have led hackers the ability to hack vehicles [5]. Due to software vulnerabilities, hackers have become able to control a vehicle from some distance away. Aside from safety and security risks, Bluetooth may pose a threat to human health. Radiation from Bluetooth has become a recent concern. Since Bluetooth uses radio transmissions that hop around the frequency, it is these "pulsed radio frequency signals" that emit radiation [7]. The rate at which the human body absorbs radiation due to Bluetooth signals is $0.23 \mathrm{~W} / \mathrm{kg}$. Comparing the Bluetooth specific absorption rate (SAR) to low SAR cellphones, in some cases the Bluetooth SAR is worse than the low rated SAR cellphone. The amount of radiation emitted is directionally proportional to the Bluetooth power class. Considering that Class I devices operate at the highest power level, the most radiation is emitted from Class I devices. Studies have shown that Class I Bluetooth devices emit similar levels of radiation as cellphones, which may ultimately contribute to DNA damage.

\section{Literature Review}

Numerous studies have been conducted to explore the applications of Bluetooth technology in the last decade. In this section, existing studies related to Bluetooth travel time technologies are summarized and sorted chronologically. But before that, examining some solutions provided by different organizations can help in understanding the mechanics of collecting travel time data by Bluetooth technology.

Many organizations provide their own approaches for measuring transportation related parameters by Bluetooth and other wireless technologies [8] [9] [10] [11]. The process of collecting data by Bluetooth sensors and software are similar. The Bluetooth software detects Bluetooth or radio signals being emitted from driver/passenger cellphones as well as vehicles. As a Bluetooth sensor detects the radio signals from approaching vehicles, Bluetooth software assigns the 
vehicle with an anonymous Mac address and records the data (time) at that instant. When the car begins to approach the second antenna, the Bluetooth software matches the anonymous Mac address with the vehicle from the first antenna and records the corresponding data. Some companies have suggested using the Bluetooth software with Wi-Fi to increase the match rate of vehicles. Without utilizing Wi-Fi, the Bluetooth software is unable to measure intersection delays [8]. Studies conducted by ITS International show that the Bluetooth software can be just as accurate as Automated License Plate Readers (ALPR) [9]. Moreover, Bluetooth systems tend to be cheaper than ALPR. Since ALPR requires cameras to be mounted in essentially every lane, the cost of camera implemented technology is more expensive than installing two Bluetooth antennas or poles. But they also conclude that, although Bluetooth detection systems seem promising, more testing is required to fully compare its benefits with current detection systems. Other Bluetooth travel time measurement systems claim that, since the Bluetooth vehicle detection rate is quite high, traffic managers are able to update travel time message signs and respond to roadway incidents much faster [10]. One of the leading global companies providing solutions in traffic management [11] note that knowing the number of stops and the durations of delays experienced by a vehicle, one may have a better estimate for the amount of carbon emissions being released into the atmosphere.

The remainder of this section describes the assessment of different researches in using Bluetooth technology for travel time measurements.

\subsection{Studies Conducted by the University of Maryland [12] [13] [14] [15] [16]}

In 2008 the University of Maryland launched a project to investigate travel times along the I-95 corridor. At the beginning, the project spanned approximately 1500 miles of freeway and 1000 miles of arterials along parts of Delaware, Pennsylvania, New Jersey, Maryland, Virginia, and North Carolina. Temporary Bluetooth scanners were installed along roadways to detect approximately $5 \%$ of vehicles within the traffic stream. To establish reliability, GPS probe vehicles were deployed to collect traffic data at the same times the Bluetooth sensors were collecting data. This experiment investigated the quality of GPS collected data against Bluetooth collected data. Researchers found that the data proved to be dependable. The collected data fell within the specifications of an average absolute speed less than $10 \mathrm{MPH}$ and speed error bias of $5 \mathrm{MPH}$. Additionally, it was found that the GPS data has a superior quality at higher speeds.

In the next few years, the University of Maryland continued their research on travel time measurement by Bluetooth sensors. The researchers conducted an experiment involving Bluetooth detection sensors to investigate the ability to estimate the specific lanes the detected vehicles are traveling in. Consequently, they have proposed an estimation technique to provide an average speed for vehicles traveling in different lanes. In 2012, The Center for Advanced Transporta- 
tion Technology at the University of Maryland provided a report regarding the research for using Bluetooth traffic detectors and their ability to be used as permanently installed travel time instruments. The installed sensors operated for 24 hours a day for the duration of almost a year. Since that, the report provides useful information for estimating the cost of operating and maintaining the Bluetooth sensors. The author, Young, notes that factors that influence detection rates include "primarily distance and proximity to the traffic stream". He specifies that the most ideal height to place Bluetooth sensors to be about 10 feet above the ground, in order to maximize detection rates. One of the biggest problems Young faced was the reliability of cellular communications. Outages and maintenance on cellular towers caused disturbances in data collection.

Haghani et al. assesses the reliability of travel time reports based on the selection of the data source and published the results of their study in 2014. In order to make a valid comparison, they use data provided by Bluetooth technology and probe data provided by a private company. However, when statistical methods were applied to the two sets of data, it was concluded that there was no significant evidence to suggest that the reliability of the Bluetooth data and the probe vehicle data were different.

This project consisted of deploying two data collection methods-GPS probe vehicles and Bluetooth technology. Relative to the GPS probe vehicles, the Bluetooth technology allowed for a direct sample of travel times and determined overall traffic travel time. The researchers for this particular project concluded that using GPS and Bluetooth provided a better overall travel time measurement experience.

\subsection{An Investigation of Bluetooth Technology for Measuring Travel Times on Arterial Roads: A Case Study on Spring Street [17]}

In 2011, Vo published his cases study on measuring travel time on arterial roads by Bluetooth technology. The author notes that the type and placement of Bluetooth sensors dictates the "quality and quantity of the data". Vo also mentions that arterials are more likely to experience "trip-chaining," where drivers stop for errands or other purposes between Bluetooth sensors, thus producing inaccurate travel times. For roads with higher traffic volume, it is suggested to use shorter sampling times. To prevent oversampling, the sensors were placed at mid-block locations, if indeed available. The first result Vo noticed from the data is that the Bluetooth technology only detected a small percentage of the total cars traveling in the detection zone, but vehicles traveling in lanes closer to the Bluetooth sensor were detected more frequently. Moreover, the author also noticed that, between the two sensors that were investigated, the heights by which the Bluetooth readers were varied at produced fluctuating results. The author found that having multiple Bluetooth readers at one station produced a higher number of total detected vehicles. Comparing the Bluetooth data to the GPS data, Vo 
found that the error between the two data sets was generally under $2 \%$.

\subsection{Studies Conducted by the University of Washington [18] [19]}

In the same year, a study published by a team of engineers and researchers at the University of Washington shows their investigation on the errors associated with Bluetooth Mac address matching for travel time measurement. Properly filtering out these modes of travel produced the traffic data regarding each mode of transportation. Additionally, the team mentions that Bluetooth sensors may take up to 10.24 seconds to detect a Bluetooth-enabled device, thus detection zones should be designed to ensure that a high-speed moving vehicle is within the detection zone long enough to be identified. After conducting experimentation, the team found that the error produced from using Bluetooth technology ranged from $4.0 \%$ to $9.4 \%$ when compared to the data collected by the ALPR cameras. The team of researchers suggests that despite this small percentage of error, the Bluetooth technology was still representative of actual conditions.

A few years later, Wang et al. at the University of Washington conducted a study to compare the error between multiple methods of travel time measurement. The modes of measurement studied in this investigation included automated license plate recognition (ALPR), Bluetooth sensors provided by a private company, and traffic data provided by a private company. The researchers sought to compare the mean absolute deviation (MAD) and the mean percent error (MPE). In order to measure such error, the team looked into mean absolute percent error (MAPE) and the root mean square error (RMSE). It was found that the Bluetooth sensors had detected significantly fewer vehicles than ALPR cameras. Consequently, after analyzing all the modes of measurement, the researchers suggest that the benefits and drawbacks of each method should be compared and contrasted (e.g., cost, operating condition, power supply) and then selected based on which method best suits the desired application.

\subsection{Studies Conducted in Canada [20] [21] [22]}

Szuch \& McDaniel's research released in 2011 summarized that transportation systems engineers in Calgary, Canada investigated Bluetooth methods to accurately measure travel times. To ensure travel time reliability, the team of researchers required a $3 \%$ detection rate of the total vehicles traveling along the route, as well as a minimum of twenty days of data collection. The engineers found that there was little variation between the two Bluetooth data sets collected.

Furthermore, Shahram describes a study described by that was conducted in Canada to determine travel time and speed recorded by Bluetooth technology. In order to determine travel time reliability, certain metrics recommended by FHWA were considered: 95\% travel time, buffer index, travel time index, and planning time index. Ultimately, it was concluded that the Bluetooth technology could produce reliable data; however, the enormous amount of data that was 
collected for a short study period made analyzing the results quite difficult. After comparing the two groups of results collected by two systems provided by different companies, the researchers suggest that there is a need for further research into crowdsourcing accuracy and the required minimum penetration rate for Bluetooth detection.

In 2014, students at McGill University published their study on investigating the ability to record traffic data using a Wi-Fi/Bluetooth detection system. Essentially, Wi-Fi works different from Bluetooth sensors. It communicates by "listening" to the signals broadcasted by Wi-Fi devices. Due to the lack of Bluetooth-enabled devices, Wi-Fi has become increasingly popular since it has a higher detection rate. The researchers note that this data would be useful in estimating the number of individuals at a bus stop, accident detection (e.g., queue length and speed), and even smart traffic apps on cellphones. Additionally, they suggest that future studies should consider activity-based modeling for urban areas, airports, campuses, and public transportation hubs. They also note, it would be useful to investigate public transportation planning as well as accident occurrence prediction.

\subsection{Studies Conducted in European Countries [23] [24] [25] [26]}

In 2011, Australian researchers published a study focusing the application of deploying Bluetooth technology for collecting traffic congestion data. Not only do the researchers consider the travel times collected by the Bluetooth sensors, but they also consider the "Duration" data-which describes the time spent by Bluetooth-enabled devices to pass through the detection range of the Bluetooth sensors. What the researchers found upon processing the data was that the $\mathrm{Du}$ ration data had an average value of about twenty seconds, providing a travel speed close to the free flow speed. Moreover, they also found that Duration increases as travel time increases. With fixed red-light signals, the Duration data was able to provide details about traffic delays, such as that travel time was heavily impacted by intersections downstream and mid-link delays. After analyzing the data, the team suggests that longer study periods should have been used to increase the sample size in order to fully understand the delays.

Researchers at the University of Portugal investigated traffic detections via Bluetooth technology by deploying two research methods and published their studies in 2013. The first method describes installing Bluetooth sensors at key points in the city, whereas the second method involves placing Bluetooth sensors within commuting cars designated to travel the route. From the data, the researchers were able to determine average travel times and speeds for rush-hour traffic. Additionally, the associated sample size was large enough to develop preliminary origin-destination matrices for the studied routes. Collecting data using the Bluetooth technology within the commuting cars provided information regarding traffic volume in areas where static Bluetooth sensors were not being used. Moreover, such information regarding the traffic volume may help traffic 
engineers and managers undercover information about drivers who carpool. The authors mentioned Portugal planned to continue using Bluetooth technology to extract reliable data regarding roadway traffic.

According to a 2015 publication in the city of Bonn, Germany, researchers looked to find a cheaper alternative to collect traffic data than the currently used systems. The researchers were hoping that Bluetooth sensors may be the solution and allow them to collect traffic information to assess the performance of current posters and billboards. The team of researchers installed the sensors in billboards that were located in close proximity to the street and free from any construction zones. The study spanned a four-week duration from the middle of July to the middle of August. Upon analyzing the data, the researchers had collected over 85,000 unique Mac addresses, which originated from over 260 different Bluetooth-enabled devices. Researchers found that after comparing the two sets of data, there was a $7 \%-10 \%$ ratio. Additionally, the data was aggregated depending on the dwelling times. From this data, the researchers were able to identify recurring Mac address throughout the duration of the study. Ultimately, the researchers found that the commuter interval information provided useful mobility patterns of commuters. However, they also soon realized the importance of selecting the right Bluetooth sensor, depending on the application of the project. The team suggests that further research should consider studying correlation coefficient for the sensors to relate similar measurements.

By implementing Bluetooth sensors in floating cars, researchers were able to collect data regarding all traffic participants, which includes motorists, bicyclists, and pedestrians. The researchers at the German Aerospace Center who created the approach called it "DYANAMIC". In order to test the approach, the researchers deployed a simulation model on a program called SUMO. From the software, the researchers were able to obtain a detection probability distribution function based on the factors that were controlled during the simulation. The team of researchers was hoping that the simulation could provide them with a detection interval of Bluetooth sensors to maximize detection and match rates. Ultimately, the researchers concluded that the detection probabilities provided by the simulation and field test provided decent results; however, they suggested that the parameters of traffic characteristics should be studied in more detail because there was some variation in the probability distributions.

\subsection{Application of Travel Time Information for Traffic Management [27]}

Day et al. at Purdue University describe their findings from conducting a study using Bluetooth technology to obtain probe vehicle data. The goal of this study was to assess characteristics of traffic flow (specifically during the Brickyard 400 race in Indiana), delays due to road construction, motorists' compliance with work zone speed limits, motorists' selection of a route during road construction, and an analysis of a signalized arterial during the winter season. The researchers suggested that Bluetooth sensors should be installed at eight feet. 


\subsection{A Methodology for Using Bluetooth to Measure Real-Time Work Zone Travel Time [28]}

Stephanie Zinner, a graduate student at Georgia Institute of Technology, developed a methodology to measure real-time travel time in a work zone on I-270 in Atlanta, Georgia. Moreover, Zinner investigates the characteristics of using different classes of Bluetooth devices for data collection. Upon analyzing the results, Zinner found that the Bluetooth sensors had a match rate between $12 \%$ and $47 \%$. Additionally, the placement of Bluetooth sensors affects the match rate indeed. Also, Zinner found that detection rates increase as speed decreases and proximity increases.

\subsection{Reliability of Bluetooth Technology for Travel Time Estimation [29]}

Araghi et al. published their investigation on the reliability of Bluetooth technology for travel time estimates in 2013. In this experiment, data collected from Bluetooth signals was compared against data collected from a GPS for test runs of 1000 trips. The researchers found that many factors influence the accuracy and reliability of Bluetooth traffic data collection. Some of these factors include the strength and speed of the Bluetooth transmitting device, the software's discovery procedure, the location of the transmitting device relative to the Bluetooth roadway sensor, and the size/shape of the detection zone. According to experimentation, the authors found that short-range Bluetooth antennas detected Bluetooth-enabled devices in a closer range to the sensor, thus producing more accurate time stamps. However, short-range antennas subsequently decrease the accuracy of penetration rates within the detection zone.

\subsection{Validation of Origin-Destination Data from Bluetooth Re-Identification and Aerial Observation [30]}

A publication of Chitturi et al. in 2014 reported how the Department of Transportation in Wisconsin (WisDOT) sought to validate two specific methods of travel time measurement. The two methods are time-lapse aerial photography (TLAP) and Bluetooth sensors. In order to compare the two methods of measurement, WisDOT collected traffic volume data so that origin-destination (OD) matrices could be generated for each method. The capture rates for the Bluetooth sensor ranged from $2.3 \%$ to $7.2 \%$, with an average of $4.4 \%$. Researchers also notice that the capture rates were bias to one particular direction of traffic. After applying the GEH (Geoffrey E. Havers) formula to the Bluetooth data, the researchers found that daily data collection from Bluetooth sensors did not provide an accurate OD matrix, but instead a combination of multiple days of data collection seemed to provide a more accurate OD matrix. The researchers suggest that the low penetration rate of Bluetooth-enabled devices may result in a low sampling rate, and thus, varying data. However, they note that Bluetooth sensors do provide satisfactory results, given the characteristics of the sensors. 


\subsection{Collecting Travel Times with Wi-Fi and Bluetooth [31]}

Mike Post, Eastern Region Director at Iteris Inc., outlines the literature associated with performing a study to retrieve travel times using $\mathrm{Wi}-\mathrm{Fi}$ and Bluetooth technology. Additionally, he outlines the possible modes of collecting data via Bluetooth-using existing cables, portable, AC-powered, and solar-powered units. Because many studies choose to use Wi-Fi in addition to Bluetooth, Post discusses the benefits of each method. He writes that Bluetooth technology allows for a continuous scan of Bluetooth signals, retrieves Mac addresses at high speeds, and also has a very high re-identification rate of Mac addresses. On the other hand, he writes that Wi-Fi continuously listens for $\mathrm{Wi}-\mathrm{Fi}$ signals, can act as hotspots, and can detect more signals (e.g., cellphones). However, Wi-Fi has a lower re-identification rate of Mac addresses and retrieves Mac addresses at a slower rate than Bluetooth. Post includes that, due to the characteristic of Wi-Fi data collection, Wi-Fi should be used where traffic is slower or has the opportunity to stop. Conversely, Bluetooth technology should be used in free flow conditions.

\subsection{Use of Travel Time, Travel Time Reliability, and Winter Condition Index Information for Improved Operation of Rural Interstates [32]}

Comparing speed sensors data to Bluetooth collected data, the state of Wyoming conducted an investigation to determine the best methodology to measure travel times on rural freeways. Researchers found that the penetration rate was extremely low on rural interstates. Additionally, upon processing the Bluetooth data, the researchers found that the Bluetooth collected travel times were longer than the speed sensors. Specifically, there was a ten-minute average difference between Bluetooth and speed sensor travel time. They note that such may be a reason due to more trucks carrying Bluetooth-enabled devices than passenger cars. Moreover, since the penetration rate of Bluetooth-enabled vehicles was found to be extremely low, the team concluded that Bluetooth technology provided unreliable results to be used in measuring travel times on rural roadways.

\section{Case Studies}

In this section, three different projects conducted by the authors at the University of Delaware, Department of Civil and Environmental Engineering are described. All these projects relate to the measurement of travel times using Bluetooth technology.

\subsection{Case study I: 2013 Bluetooth Data Collection on Route 1- The State of Delaware, USA [33]}

In 2013, a study was launched in Delaware to observe traffic behavior at designated locations along Route One. This study was the DelDOT's (Delaware Department of Transportation) first attempt of using Bluetooth technology for travel time measurements. The University team assisted the DelDOT in processing 
data and conducting analyses.

A total of six Bluetooth sensors (Figure 1) were installed at the following locations (relative to Route One)-DE 30, DE 16, Five Points, Collins Street, DE 26, and DE 54. More specifically, the identification of each of the sensors, respectively, were SBT0003, SBT0004, SBT0001, SBT0002, SBT0005, and SBT0006. The sensors were deployed for data collection on four separate days-Sept. 25, 2013 (6:30 AM-9:30 AM), Sept. 26, 2013 (3:30 PM-6:30 PM), Oct. 1, 2013 (6:30 AM-9:30 AM), and Oct. 22, 2013 (3:30 PM-6:30 PM). The three-hour periods of data collection were considered to be the peak hours of data collection. Each Bluetooth reader had a scan length of 5.12 seconds. On the four days of data collection, the Bluetooth sensors collected traffic data in both directions of traffic. To validate the Bluetooth data, travel times derived from GPS probe vehicles were used for comparisons.

The data from all six Bluetooth sensors was separated into four Excel spreadsheets based on the date the data was collected. It was ensured that all reported detection times were converted into standard time for processing simplicity. Each Mac address was individually analyzed for a match between Bluetooth sensors. If the Mac address was recognized by more than one sensor, the travel time was calculated by taking the difference between time stamps. An example of the travel time calculation is provided in Figure 2. Once all the individual travel times had been calculated, the average travel time for a particular segment on a particular day could be generated to compare with the GPS travel times.

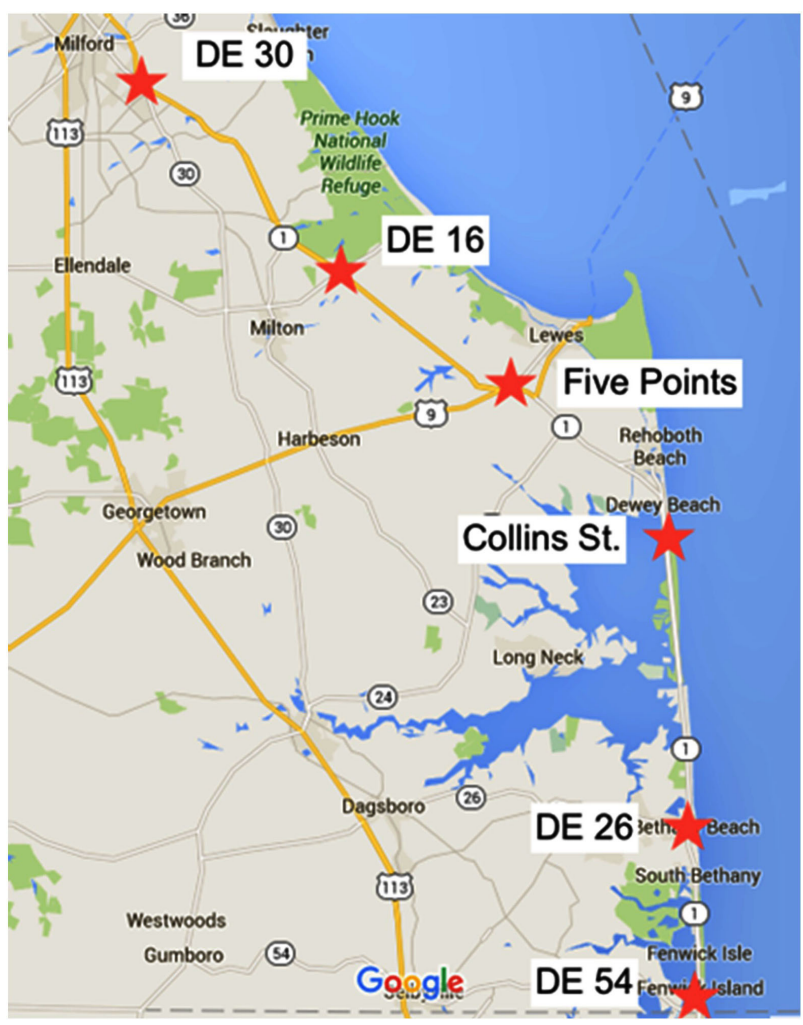

Figure 1. The map of Bluetooth Sensors, 2013 [33]. 


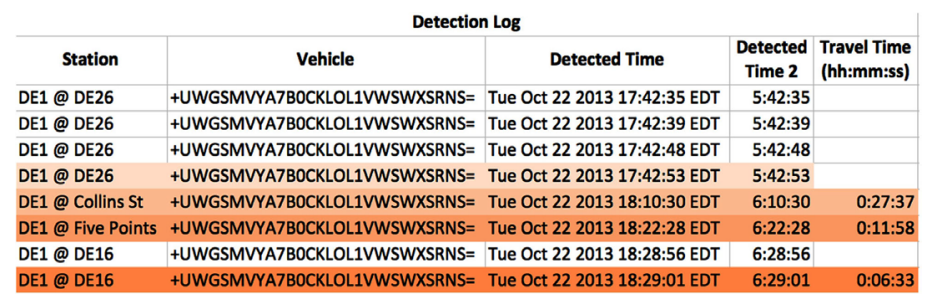

Figure 2. Example of calculating travel time based on Bluetooth data [33].

The total number of detected vehicles for this study was 3399. The detection rates of all roads were ranged from $5 \%$ to $36 \%$. For each daily observation period, the average travel time collected Bluetooth and GPS probe vehicle methods were compared. The GPS travel time data was also used for filtering out the outliers of Bluetooth data. Bluetooth travel times that were three times greater than the average GPS travel time for a particular segment were excluded from the calculation of average travel time reported by Bluetooth technology. Additionally, to illustrate the importance of filtering, calculations were repeated for the data after Bluetooth travel times, two times greater than the average GPS travel times were excluded as outliers. Since standard deviation and coefficient of variance are both common statistical measurements that provide the information for how "spread out the data points are", they were also calculated and analyzed in this study. The data for each observation period-each three hours, was analyzed separately. The following Table 2 and Table 3 show examples of how the resulting data was presented.

As demonstrated by the results, Bluetooth sensors can provide satisfactory results of travel time measurement. However, upon manually analyzing the data, a few interesting details were noticed. In this analysis, last-to-last matching was used; that if a sensor detected a vehicle more than once, the last recorded detection would be used in the travel time calculations. As the travel times were being calculated, it was noticed that in several situations a significant phenomenon kept repeating. More specifically, a vehicle would be detected at a sensor at some time $\mathrm{X}$ and would be detected at the following sensor at a much later time Y. Consequently, longer travel times would be produced from these situations. Despite the attempt to filter out any outliers in the data, some of these longer travel times were still within the error threshold and thus, were accounted in the calculated average travel time for each segment. When the filtering procedure was adjusted to eliminate Bluetooth travel times two times greater than the average GPS travel time (rather than three times greater), many of the average Bluetooth travel times changed considerably. In some cases, the average Bluetooth travel time was decreased by as much as one minute when compared to the previously calculated Bluetooth travel time (times that filtered out data three times greater than the GPS average). Further research and investigations into Bluetooth travel time data collection may help develop a specific methodology for determining "ground truth" data. The match rate for each day of data collection was also calculated [33]. During data processing, it was noticed that some sensors matched more vehicles than the other sensors, which may be a result of reduced traffic density. 
Table 2. Example of travel time data table regarding Bluetooth and GPS methods [33].

\begin{tabular}{lcccccc}
\hline & \multirow{2}{*}{ Direction } & SR 30/ & SR 16/ & Five Points/ & Collins St./ & SR 26/ \\
& & SR 16 & Five Points & Collins St. & SR 26 & SR54 \\
Coefficient of Variance I & SB & $6.1 \%$ & $18.1 \%$ & $22.1 \%$ & $12.0 \%$ & $32.9 \%$ \\
Coefficient of Variance II & SB & $6.1 \%$ & $7.6 \%$ & $10.4 \%$ & $12.0 \%$ & $21.8 \%$ \\
Coefficient of Variance I & NB & $11.9 \%$ & $6.6 \%$ & $35.0 \%$ & $27.8 \%$ & $31.5 \%$ \\
Coefficient of Variance II & NB & $11.9 \%$ & $6.6 \%$ & $20.2 \%$ & $5.0 \%$ & $15.9 \%$ \\
\hline
\end{tabular}

Table 3. Example of coefficient of variance table [33].

\begin{tabular}{ccccccc}
\hline & Direction & SR 30/ & SR 16/ & Five Points/ & Collins St./ & SR 26/ \\
& SR 16 & Five Points & Collins St. & SR 26 & SR54 \\
\hline Distance & Miles & 9.4 & 6.7 & 7.2 & 10.4 & 6 \\
GPS Average & SB & $09: 04$ & $07: 14$ & $11: 42$ & $10: 55$ & $08: 12$ \\
Bluetooth Average I & SB & $09: 39$ & $07: 22$ & $14: 12$ & $11: 29$ & $10: 41$ \\
Standard Deviation I & SB & $00: 57$ & $01: 31$ & $03: 12$ & $01: 36$ & $03: 42$ \\
Bluetooth Average II & SB & $09: 39$ & $07: 11$ & $13: 26$ & $11: 29$ & $09: 56$ \\
Standard Deviation II & SB & $00: 57$ & $00: 54$ & $01: 38$ & $01: 36$ & $02: 08$ \\
GPS Average & NB & $09: 03$ & $06: 40$ & $13: 29$ & $10: 53$ & $07: 46$ \\
Bluetooth Average I & NB & $09: 00$ & $07: 02$ & $18: 32$ & $11: 36$ & $09: 56$ \\
Standard Deviation I & NB & $01: 07$ & $00: 46$ & $06: 40$ & $03: 16$ & $03: 01$ \\
Bluetooth Average II & NB & $09: 00$ & $07: 02$ & $15: 32$ & $11: 00$ & $09: 14$ \\
Standard Deviation II & NB & $01: 07$ & $00: 46$ & $03: 09$ & $00: 55$ & $01: 45$ \\
\hline
\end{tabular}

\subsection{Case Study II: 2015 GPS Probe Vehicle Data and Bluetooth Sensor Data Comparison-The State of Delaware, USA [33]}

In 2015, a more extended study of using and evaluating Bluetooth technology for travel time measurements was conducted. The investigators (the authors) were trying to understand and experience the advantages and disadvantages of using Bluetooth technology for travel time measurements, and observe first-hand how such things as the position of the Bluetooth sensors, trip-chaining, oversampling and other risk factors can impact the results.

GPS probe vehicles and Bluetooth sensors were both deployed to collect travel time data along US 113. GPS probe vehicles were equipped with navigation equipment to detect and record locations and timestamps as the probe vehicle traversed the route. Using GPS signals, the equipment could pinpoint the exact location of the vehicle and determine when the vehicle has crossed predetermined checkpoints. Many of these checkpoints correspond to the exact same locations as the deployed Bluetooth sensors; thus, a comparison between the two methods of measurement could be made. Once the probe vehicle crosses a checkpoint, the timestamp is recorded on the navigation device and travel times can be determined between checkpoints. Figure 3 displays locations of GPS probe vehicle checkpoints and Bluetooth sensors. 


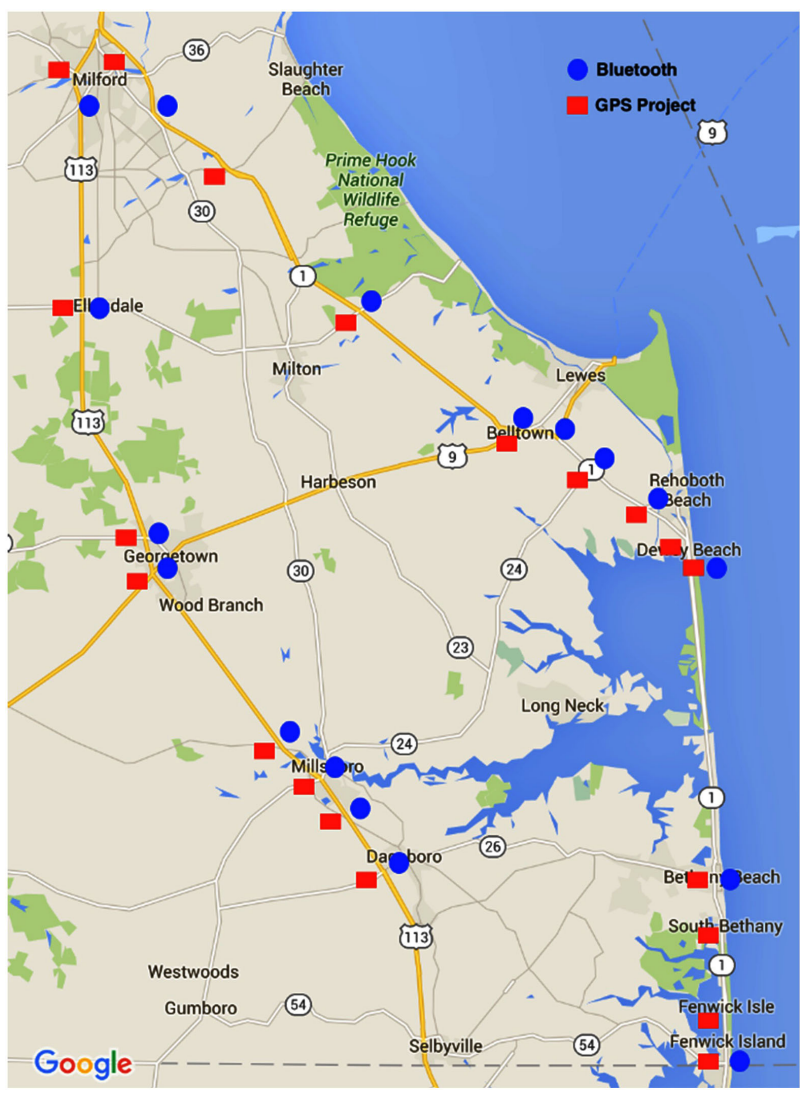

Figure 3. The map of Bluetooth Sensors and GPS Probe Vehicle Checkpoints, 2015 [33].

Upon receiving the data for comparison, it is important to note that an outside source had previously processed and reduced the Bluetooth data with unknown algorithms and techniques. The GPS data had also been processed by the research team and average travel times were used for comparison. The Bluetooth sensor data was collected in fifteen-minute intervals. However, the GPS probe vehicle data was collected during the marked peak hours and the GPS travel times corresponded to the timestamp at which the vehicle crossed the checkpoint. Consequently, the time at which the average GPS travel times were collected may not exactly match the times at which the Bluetooth sensors collected travel time data. To account for this small difference, GPS travel times were "rounded up" to the closest fifteen-minute interval to match the Bluetooth data. For example, if the GPS timestamp at which the vehicle crossed the checkpoint occurred at 4:52 PM, the Bluetooth average travel time that was used in the comparison was collected at 5:00 PM. Table 4 shows an example of travel time comparison between Bluetooth and GPS data.

The results show that travel times reported by the GPS probe vehicles and Bluetooth sensors are close. Some of the times are only within seconds of each other, others close to a minute. Additionally, since the GPS and Bluetooth travel times were not collected at the exact same time as each other, there may be some variation in the results displayed. Moreover, it is important to note that the 
Table 4. Example of travel time data comparisons between Bluetooth and GPS methods [33].

\begin{tabular}{cccccccc}
\hline Segment & $\begin{array}{c}\text { Bluetooth } \\
\text { Sensor }\end{array}$ & Direction & Distance & Count & Timestamp & $\begin{array}{c}\text { GPS Time } \\
(\mathrm{sec})\end{array}$ & $\begin{array}{c}\text { Bluetooth Time } \\
(\mathrm{sec})\end{array}$ \\
\hline SR 24 - US 9 & SBT0007 to SBT0009 & NB & $8.66 \mathrm{mi}$ & 9 & $4: 49 \mathrm{PM}$ & 631 & 570 \\
SR 20 East - SR 24 & SBT0008 to SBT0007 & NB & $1.60 \mathrm{mi}$ & 4 & $5: 00$ PM & 126 & 127 \\
US 9 - SR 404 & SBT0009 to SBT0010 & NB & $1.17 \mathrm{mi}$ & 6 & $5: 02$ PM & 97 & 114 \\
SR 404 - SR 16 & SBT0010 to SBT0031 & NB & $7.98 \mathrm{mi}$ & 10 & $5: 09$ PM & 454 & 490 \\
\hline
\end{tabular}

reported Bluetooth sensors tend to overestimate the travel times. This trend may be a result of the unknown locations of the vehicles detected by the Bluetooth sensors within the detection zone. This characteristic of Bluetooth sensors may be enough to create a bias in the travel times to overestimate.

\subsection{Case Study III: 2019 Bluetooth Travel Time Data Information Extraction-The State of Delaware, USA}

Starting in 2016, the Department of Civil and Environmental Engineering, at the University of Delaware, started to use Bluetooth data for state-wide travel time measurement as an alternative to GPS probe vehicle data collection approach. Having gained experience from last two case studies, the authors continued analyzing collected data, and working with the state officials closely to see how the results can be incorporated into the overall congestion management system. Advantages and disadvantages of using Bluetooth technology by itself as opposed to using Bluetooth with GPS and other technologies were clearly communicated to the professional staff in the state. The mechanics of collecting data by GPS data was by driving probe vehicles through target roadways based on standards set by the Federal Highway Administration (FHWA) [34] in critical periods of a day. Each run of data collection had to be accomplished by a group of students.

The process of Bluetooth travel time data collection adopted by the University of Delaware was similar to the aforementioned approaches used by different teams. Figure 4 shows how the University collected travel time data by Bluetooth sensors. The method of how to obtain one travel time record was introduced in section 3.1. But that process wasn't accomplished by the University of Delaware. The data that University of Delaware received was stored in an Excel file and had three sheets: 15-min average travel time, 15-min median travel time, and 15-min observation number. The first sheet stored the average travel time data for each "segment" (a road section between two consecutive Bluetooth sensors) and each 15-minute observation interval. The second sheet showed the median of travel time for each "segment" and each 15-minute interval. The last sheet stored the number of vehicles that was detected and matched by two consecutive Bluetooth sensors in each 15-minute observation interval. The three sheets were used to calculate different measures that DelDOT staff requested including monthly and annual average travel times for the three counties and for the State of Delaware. The analysis results for Fall 2016, Summer and Fall 2017 
were reported to the DelDOT [35] [36] [37].

In 2019, the Bluetooth travel time data analysis team at the University of Delaware was requested to extract data and information in order to comply with the new FHWA standards [38] [39] [40]. The objectives included 1) calculating the planning time index; 2) determining appropriate speed limits based on the 85th percentile operational speed, and 3) identifying and categorizing congestions and causes.

For the first and the second objectives, different percentiles of travel time data were required. Planning time index equals to the ratio of the 95th percentile travel time to free-flow travel time [38]. The way to calculate free-flow travel time varies. FHWA suggests using travel time at the 85th percentile of off-peak operational speeds as free-flow travel time for the Urban Congestion Report [41]. Figure 5 shows an example of the outcome of the 85th percentile operational speed analysis. Both objectives required percentiles of different groups of travel time data. But the data that the authors received only provided the preprocessed average and median values of travel time and observation number for each time interval. So, taking the percentiles of those averages and medians could not provide the exact percentiles of the entire population.

Comparing the data collection processes by GPS probe vehicles and Bluetooth sensors, it's easy to see that one drawback of Bluetooth method is the difficulty on determining the causes of congestion. As mentioned, during the collection of travel time data by GPS probe vehicles, students were also asked to record the causes of congestions. But obviously the Bluetooth sensors were not capable of collecting such information.

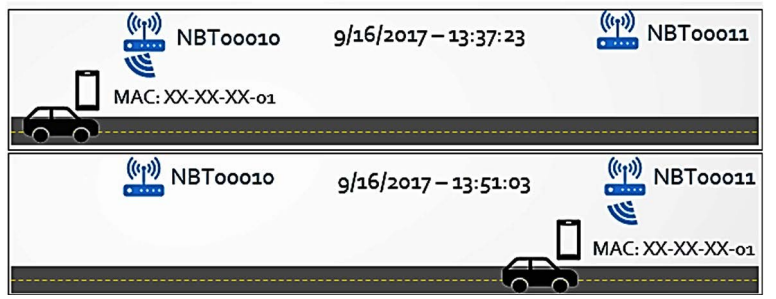

Figure 4. Collecting travel time data by Bluetooth sensors [42].

\begin{tabular}{|c|c|c|c|c|c|c|c|c|c|c|}
\hline Segment & Route Name & Route Description & $\begin{array}{c}\text { Direction of } \\
\text { Traffic }\end{array}$ & County & $\begin{array}{l}\text { Length } \\
\text { (Mile) }\end{array}$ & \begin{tabular}{|c|}
$\begin{array}{c}\text { Weighted } \\
\text { Average } \\
\text { Speed Limit } \\
\text { (mph) }\end{array}$ \\
\end{tabular} & $\begin{array}{l}\prod_{\text {at Speed }} \\
\text { Limit (min) }\end{array}$ & \begin{tabular}{|c}
$\begin{array}{c}\text { Overall 85th } \\
\text { Perct Spd } \\
\text { (mph) }\end{array}$ \\
\end{tabular} & \begin{tabular}{|c|}
$\pi$ at Overall \\
85th Perct \\
Spd (min)
\end{tabular} & 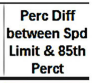 \\
\hline KBT0001-КBTO008 & SR 1 & Toll Plaza to Exit 104 & NB & Kent & 2.33 & 58.33 & 2.40 & 73.23 & 1.912 & $25.55 \%$ \\
\hline KBT0001-КBTO025 & SR 1 & Toll Plaza to S Bay Rd & SB & Kent & 3.35 & 51.00 & 3.94 & 66.99 & 3.001 & $31.35 \%$ \\
\hline КВто001-КВто029 & SR 1 & Toll Plaza to Exit 98 & SB & Kent & 1.63 & 49.93 & 1.96 & 67.09 & 1.457 & $34.36 \%$ \\
\hline КВТо002-КВто003 & SR 1 & SR 9 to Bowers Beach Rd & SB & Kent & 4.47 & 55.00 & 4.87 & 65.58 & 4.088 & $19.24 \%$ \\
\hline KBTO002-КBт0015 & SR 1 & Exit 91 to Exit 97 & NB & Kent & 4.39 & 65.00 & 4.05 & 68.43 & 3.850 & $5.27 \%$ \\
\hline KBT0002-KBTO025 & SR1 & $\begin{array}{l}\text { SR9 and Puncheon Run } \\
\text { Connector }\end{array}$ & NB & Kent & 3.71 & 50.85 & 4.38 & 57.52 & 3.874 & $13.11 \%$ \\
\hline Kвто003-КВт0002 & SR 1 & Bowers Beach Rd to SR 9 & NB & Kent & 4.48 & 55.00 & 4.88 & 64.87 & 4.140 & $17.94 \%$ \\
\hline КВто003-КВт0013 & SR 1 & Bowers Beach Rd to SR 12 & SB & Kent & 1.85 & 55.00 & 2.02 & 65.81 & 1.691 & $19.65 \%$ \\
\hline КВТо004-КВт0010 & Us 113 & Rd 409 to SR 14 & SB & Kent & 3.04 & 45.00 & 4.06 & 50.46 & 3.617 & $12.13 \%$ \\
\hline KBTO004-KBT0013 & SR 1 & $\mathrm{Rd} 409$ to $\mathrm{SR} 12$ & NB & Kent & 4.48 & 55.00 & 4.88 & 66.87 & 4.017 & $21.58 \%$ \\
\hline KBTO004-SBTO003 & SR 1 & Rd 409 to Rd 206 & SB & Kent & 5.19 & 54.78 & 5.68 & 70.42 & 4.421 & $28.55 \%$ \\
\hline
\end{tabular}

Figure 5. Example of the 85th percentile operational speed analysis outcome. 


\section{Issues Related to Using Bluetooth Sensors for Collecting Travel Time Data}

After reviewing existing studies in the literature and the three case studies, some issues were unveiled when using Bluetooth sensors for collecting travel time data. A summary of the issues is presented below.

\subsection{Unknown Location of Detected Vehicle within the Detection Zone}

By the nature of Bluetooth technology, Bluetooth used for travel time data collection leads to an enormous amount of data to be processed. Despite algorithms and software used to analyze the data, it is always good to have a first-hand review of sections of the data (if not all of the data). Consequently, the dense data makes data processing very time consuming.

\subsection{Extremely Dense Data Processing}

Since Bluetooth sensors rely on cellular communications and outside power sources (unless the sensor is solar or installed in a traffic cabinet), complications may arise with the reliability of the equipment. Heavy storms or strong winds may knock cellphone towers down which may result in communication disruptions in the sensors.

\subsection{Communications/Power Supply Complications during Sensor Deployment}

Since Bluetooth sensors are unable to exactly pinpoint the location of the vehicle when it is detected in the detection zone, a few seconds may be overestimating or underestimating the Bluetooth reported travel time. (In many cases Bluetooth travel times are overestimated when compared to other data.)

\subsection{Oversampling}

The location of the Bluetooth sensor relative to the roadway is extremely important. Bluetooth sensors placed at intersections typically detect the same vehicle multiple times, leading to an excess amount of data. Sensors deployed at mid-block locations tend to minimize the number of detections of a vehicle in one passing.

\subsection{Unable to Determine Traffic Volume}

Again, by the characteristics of how Bluetooth works as travel time measurement, this method of measurement is unable to determine the total volume of traffic during deployment. Consequently, it is unknown whether the resulting data provides a strong sample of the population. Supplemental methods must be used with Bluetooth sensors to account for traffic volume.

\subsection{Trip-Chaining}

Trip-chaining is the term used to describe when drivers stop for errands or other 
purposes between Bluetooth sensors. More common to be problematic on arterials, trip-chaining can result in misleading or inaccurate travel times if not removed from the data prior to processing.

\subsection{Low Detection/Match Rates}

Bluetooth sensors and their ability to serve as a method used for travel time measurement rely on drivers and passengers carrying at least one Bluetooth-enabled device in order to detect the vehicle. Although detection rates have improved in recent years, many drivers still do not have or do not enable Bluetooth while driving. Low detection rates may provide insufficient data for a proper data analysis.

\subsection{No Standard form of Analysis}

Data processing that has been completed in studies and research of Bluetooth sensors all implement their own data processing techniques. There is no formal standard to process Bluetooth travel time data. As a result, there may be variations in the calculated travel times due to how the data was filtered and what statistical algorithms were used.

\subsection{Limited Information Extraction}

The information extraction results based on Bluetooth travel time data rely, to a large extent, on how the data was pre-processed. As mentioned, some information, such as 85th percentile, have to be extracted from the raw Bluetooth data. Otherwise, the accuracy cannot be guaranteed.

\subsection{Difficulty of Determining Reasons for Delay}

As mentioned in the case studies, since the Bluetooth data can only provide travel time data, it has become a challenge for researchers to derive meaningful congestion information, especially the causes of congestions and delays. Sometimes, the researchers need multiple types of information from different sources to understand the reasons for congestion and delay, thus making the overall process more expensive and less reliable than existing methods.

\section{Summary, Conclusions and Recommendations}

\subsection{Summary}

Bluetooth has significantly improved and been adopted to meet higher applications than ever imagined twenty years ago. Emerging into the market as a new and easier method for users to transfer data and synchronize files, Bluetooth has become one of the most innovative techniques used today for travel time measurement. The principle characteristics of the technology allow Bluetooth to be a simple alternative to current travel time measurement methods. Bluetooth travel time sensors work by transmitting a signal, within a short-range, and waiting for a response from any Bluetooth-enabled device in the detection zone. If the Blu- 
etooth sensor receives a signal back from a Bluetooth-enabled device, the Mac address of the device is anonymously recorded, as well as the timestamp at which the device was detected.

With any method used for travel time measurement come benefits and drawbacks associated with that method, including Bluetooth sensors. Traffic managers and engineers have typically praised the cost-effectiveness of Bluetooth sensors. Compared to ALPR cameras and even speed sensors, Bluetooth sensors have much lower installation, maintenance, and operating costs. Unlike ALPR cameras, there is no requirement to have multiple Bluetooth sensors deployed in each lane, thus Bluetooth equipment costs already begin at a fraction of the price when compared to ALPR camera costs. It is the ability of one Bluetooth sensor to collect traffic data from all travel lanes that has continuously attracted traffic managers. The resulting travel time produced from Bluetooth sensor data has also been extremely useful for developing origin-destination matrices, peak hour and congestion graphs, and evaluating traffic signal intersection performance. Comparing to GPS probe vehicle technology, Bluetooth is able to collect 24/7. Such advantage can help traffic management agencies to react in a timely manner. However, as a new method to travel time measurement, a lot of issues need mitigations when deploying Bluetooth technology. These issues were specifically outlined in the body of this paper.

After analyzing previous Bluetooth sensor case studies, literature, and research, particular trends were noticed between the interaction of Bluetooth sensors and passing vehicles. Case study results have demonstrated that vehicles are more likely to be detected at slower speeds than at high speeds; however, oversampling may occur in such cases. Furthermore, studies that investigated the comparison between Bluetooth traffic data collected from stationary sensors and sensors placed in probe vehicles yielded interesting results. Probe vehicles containing Bluetooth readers were found to have a significant increase in vehicle match rate when compared to stationary Bluetooth sensors, suggesting that data collection could be more accurate in probe vehicles. In addition, case study research discovered that Bluetooth travel time analysis should rely on the raw or non-processed data. So, it requires the researchers to pay more attention to data filtering and initializing before extracting accurate information.

\subsection{Conclusions}

Bluetooth technology used for travel time measurement demonstrates many unique benefits and drawbacks. Easy to implement, Bluetooth sensors have been praised for their simplicity. However, drawbacks like low detection rates, inability to count traffic volume, discrepancies in the detection zone, dependency on power supply and cellular towers, oversampling and excessive amount of data to be processed, necessity of access to raw data has made researchers wary of the Bluetooth technology. Additionally, the phenomenon of trip-chaining has made it difficult for researchers to determine valid travel times. Comparing to other 
methods, Bluetooth technology's difficulty in collecting congestion information also challenges researchers. Moreover, since there is no standard for Bluetooth data processing, case study results may vary from each other. As a relatively new technology, complying the data analyzing results with the data reporting standards is also crucial for the applications of Bluetooth technology. Researchers suggest that decision makers looking to use Bluetooth sensors for travel time measurement should clearly identify the goal of the study and determine if the possible disadvantages of the Bluetooth sensors outweigh the benefits for a particular application. Moreover, after reviewing existing studies and case studies, only eight of those studies deemed Bluetooth sensors acceptable without any precaution or hesitation. So, further studies are necessary for investigating and improving the travel time measurement by Bluetooth technology, and the overall conclusion of the authors for this technology is that the Bluetooth technology by itself is not a proper tool for travel time measurements. However, several studies highlighted that integrating Bluetooth traffic data with other types of data, such as data collected by ALPR technology, or GPS, can help researchers to achieve more accurate and comprehensive measurements on travel time information. Such conclusion coincides with the experience gained by the authors.

\subsection{Recommendations}

This study revealed the advantages and disadvantages of the Bluetooth technology for travel time measurement. The disadvantages are serious enough to cause unreliable and inaccurate data and information. It is recommended that more studies be conducted to specifically see how each one of the aforementioned drawbacks can be fixed. Furthermore, at the present time, if a transportation agency is interested in using Bluetooth technology for travel time measurements, the users need to have a specific plan of study and make sure that the shortcomings and disadvantages of Bluetooth method do not outweigh the application for which it is intended. The authors strongly recommend conducting small studies that include accuracy, and reliability analysis, as well as comparison with existing methods before large-scale investments in Bluetooth technology is made.

\section{Acknowledgements}

The authors express sincere gratitude for having received data, information, and technical assistance from the professional staff at the Delaware Department of Transportation (DelDOT), the University of Delaware Center for Transportation Research, and Rybinski Engineering.

\section{Conflicts of Interest}

The authors declare no conflicts of interest regarding the publication of this paper. 


\section{References}

[1] Wikipedia.org, Bluetooth Special Interest Group. https://en.wikipedia.org/wiki/Bluetooth_Special_Interest_Group

[2] Bourque, B. (2014) This Is How Bluetooth Works, and No, It's Not by Magic. https://www.digitaltrends.com/mobile/how-does-bluetooth-work/

[3] Bluetooth Insight (2008) Bluetooth Power Classes. http://bluetoothinsight.blogspot.com/2008/01/bluetooth-power-classes.html

[4] Mohankumar, D. (2008) Bluetooth Technology. https://www.engineersgarage.com/contributions/bluetooth-technology/

[5] Steinberg, J. (2015) Why Your Bluetooth Devices Aren't as Secure as You Think. https://www.inc.com/joseph-steinberg/are-your-bluetooth-devices-secure-maybe-n ot.html

[6] Walsh, S., Wan, J. and Sadlier, A. (2019) Technology Survey: Bluetooth Security. http://ntrg.cs.tcd.ie/undergrad/4ba2.05/group15/

[7] Burrell, L. (2014) Bluetooth What You Will Learn Nowhere Else-Is It Really Dangerous? http://www.electricsense.com/1010/bluetooth-what-you-will-learn-nowhere-else--is-it-really-dangerous/

[8] Sensys Network (2015) Arterial Solutions: Travel Time-Bluetooth and Wi-Fi. https://www.sensysnetworks.com/products/senstraffic\#sensid

[9] ITS International (2013) Bluetooth and Wi-Fi Offer New Options for Travel Time Measurement.

http://www.itsinternational.com/categories/detection-monitoring-machine-vision/f eatures/bluetooth-and-wi-fi-offer-new-options-for-travel-time-measurements/

[10] Veovo (2018) BlipSystem: Road Traffic. https://blipsystems.com/traffic/

[11] Siemens (2014) Precise Measurement and Intelligent Processing of Travel Time Data.

https://www.mobility.siemens.com/mobility/global/SiteCollectionDocuments/en/ro ad-solutions/urban/infrastructure/travel-time-measure-en.pdf

[12] Haghani, A., Hamedi, M. and Farokhi Sadabadi, K. (2009) Summary Report for I-95 Corridor Coalition Vehicle Probe Project: Validation of INRIX Data July-September 2008.

[13] Zoto, J., La, R.J., Hamedi, M. and Haghani, A. (2011) Estimation of Average Vehicle Speeds Traveling on Heterogeneous Lanes Using Bluetooth Sensors. 2012 IEEE Vehicular Technology Conference, Quebec City, 3-6 September 2012, 1-5. https://doi.org/10.1109/VTCFall.2012.6399146

[14] Young, S.Y. (2012) Bluetooth Traffic Detectors for Use as Permanently Installed Travel Time Instruments. The University of Maryland, College Park, MD.

[15] Haghani, A., Zhang, Y. and Hamedi, M. (2014) Impact of Data Source on Travel Time Reliability Assessment. The University of Maryland, College Park, MD.

[16] Vo, T. (2011) An Investigation of Bluetooth Technology for Measuring Travel Times on Arterial Roads: A Case Study on Spring Street. Georgia Institute of Technology, Atlanta, GA.

[17] Young, S. (2015) Use of Probe and Bluetooth Data for Arterial Performance Measures in the I-95 Corridor Coalition. The University of Maryland, College Park, MD.

[18] Malinovskiy, Y., Wu, Y., Lee, U. and Wang, Y. (2011) Error Modeling and Analysis for Travel Time Data Obtained from Bluetooth Mac Address Matching. The Uni- 
versity of Washington, Seattle, WA.

[19] Wang, Y., Araghni, B., Malinovskiy, Y., Corey, J. and Cheng, T. (2014) Error Assessment for Emerging Traffic Data Collection Devices. Washington State Department of Transportation, Olympia, WA.

[20] Szuch, C. and McDaniel, Z. (2011) Measuring Goods Movement Travel Time and Reliability: Proposed Methodology. Transportation Association of Canada, Edmonton.

[21] Tahmasseby, S. (2012) Traffic Data: Bluetooth Sensors vs. Crowdsourcing-A Comparative Study to Calculate Travel Time Reliability in Calgary, Alberta, Canada. Journal of Traffic and Transportation Engineering, 3, 63-79. https://doi.org/10.17265/2328-2142/2015.02.001

[22] Lesani, A., Jackson, S. and Miranda-Moreno, L. (2014) Towards a WIFI-Bluetooth system for Traffic Monitoring in Different Transportation Facilities. McGill University, Montreal.

[23] Tsubot, T., Bhaskar, A., Chung, E. and Billot, R. (2011) Arterial Traffic Congestion Analysis Using Bluetooth Duration Data. Australasian Transport Research Forum 2011 Proceedings, Adelaide.

[24] Filgueriras, J., Rossetti, R., Kokkinogenis, Z., Ferreira, M., Olaverri-Monreal, C., Paiva, M., Tavares, J. and Gabriel, J. (2013) Sensing Bluetooth Mobility Data: Potentials and Applications. In: de Sousa, J. and Rossi, R., Eds., Computer-Based Modelling and Optimization in Transportation. Advances in Intelligent Systems and Computing, Springer, Cham, 419-431. https://doi.org/10.1007/978-3-319-04630-3_31

[25] Mueller, M., Schulz, D., Mock, M. and Hecker, D. (2016) Detecting Mobility Patterns with Stationary Bluetooth Sensors. 8th AGILE Conference on Geographic Information Science, Lisbon, Portugal.

[26] Gurczik, G. (2016) Bluetooth-Based Floating Car Observer: Model Evaluation Using Simulation and Field Measurements. German Aerospace Center (DLR) Institute of Transportation Systems.

[27] Day, C., Wasson, J., Brennan Jr., T. and Bullock, D. (2012) Application of Travel Time Information for Traffic Management. The Purdue University, West Lafayette, IN. https://doi.org/10.5703/1288284314666

[28] Zinner, S. (2012) A Methodology for Using Bluetooth to Measure Real-Time Work Zone Travel Time. The Georgia Institute of Technology, Atlanta, GA.

[29] Namaki Araghi, B., Hammershoj Oleson, J., Krishnan, R. and Lahrmann, H. (2013) Reliability of Bluetooth Technology for Travel time Estimation. Journal Intelligent Transportation Systems. Technology, Planning, and Operations, 19, 240-255. https://doi.org/10.1080/15472450.2013.856727

[30] Chitturi, M., Shaw, J., Campbell, J. and Noyce, D. (2014) Validation of Origin-Destination Data from Bluetooth Reidentification and Aerial Observation. Transportation Research Record Journal of the Transportation Research Board, 2430, 116-123. https://doi.org/10.3141/2430-12

[31] Post, M. (2014) Collecting Travel Times with Wi-Fi and Bluetooth.

[32] Milliken, E. and Young, R. (2015) Use of Travel Time, Travel Time Reliability, and Winter Condition Index Information for Improved Operation of Rural Interstates. Mountain-Plains Consortium.

[33] Partridge, K. and Faghri, A. (2016) Bluetooth Technology. The University of Delaware, Newark, DE. 
[34] Office of Highway Information Management, FHWA, U.S. DOT (1998) Travel Time Data Collection Handbook. FHWA, U.S. DOT.

[35] Faghri, A., Mohammadiziazi, R., Nerwinski, Z. and Yuan, D. (2016) Processing of DelDOT Bluetooth Data for Travel Time Measurements-Fall 2016. Delaware Center for Transportation, Newark, DE.

[36] Faghri, A., Yuan, D., Wang, Y. and Li, M. (2018) Processing of DelDOT Bluetooth Data for Travel Time Measurements-Summer 2017. Delaware Center for Transportation, Newark, DE.

[37] Faghri, A., Yuan, D., Wang, Y. and Li, M. (2019) Processing of DelDOT Bluetooth Data for Travel Time Measurements-Fall 2017. Delaware Center for Transportation, Newark, DE.

[38] Federal Highway Administration, U.S. Department of Transportation (2006) Travel Time Reliability: Making It There on Time, All The Time. https://ops.fhwa.dot.gov/publications/tt_reliability/TTR_Report.htm

[39] Federal Highway Administration, U.S. Department of Transportation (2013) Methods and Practice for Setting Speed Limits: An Informational Report.

[40] Federal Highway Administration, U.S. Department of Transportation (2005) Traffic Congestion and Reliability: Trends and Advanced Strategies for Congestion Mitigation. https://ops.fhwa.dot.gov/congestion_report/executive_summary.htm

[41] Federal Highway Administration, U.S. Department of Transportation (2015) The Urban Congestion Report (UCR): Documentation and Definitions. https://ops.fhwa.dot.gov/perf_measurement/ucr/documentation.htm

[42] Federal Highway Administration, U.S. DOT (2009) Speed Concepts, Information Guide. U.S. DOT. 